\title{
Computerized Maintenance Management Systems in SMEs: a survey in Italy and some remarks for the implementation of Condition Based Maintenance
}

\author{
Luca Fumagalli*, Marco Macchi*, Mario Rapaccini** \\ *Department of Management, Economics and Industrial Engineering, \\ Politecnico di Milano, Piazza Leonardo da Vinci 23, 20133, Milano \\ Italy(email: luca1.fumagalli@polimi.it; marco.macchi@polimi.it) \\ ** Dipartimento di Energetica 'Sergio Stecco', Università Degli Studi di Firenze, \\ Via Santa Marta, 3-50139, Firenze, Italy (email: mario.rapaccini@unifi.it)
}

\begin{abstract}
Condition Based Maintenance (CBM) is considered one of the most relevant policies for the improvement of maintenance management for the next future. The CBM consists of a maintenance program that recommends maintenance actions based on the information collected through condition monitoring (Jardine, 2006). With the rapid development of computer and advanced sensor technologies, data acquisition has become more powerful and less expensive, making condition monitoring more affordable. However, this seems not to be enough. Condition monitoring data are only the measurements related to the health condition of the physical asset. Asset life cycle management requires more than that: for example, a registry of event data for the physical asset (information on what happened, e.g., installation, breakdown, overhaul, etc., and what was done, e.g., minor repair, preventive maintenance, oil change, intervention from a third party, etc.), which is normally handled in Computerized Maintenance Management Systems (CMMS), cannot be overlooked when doing CBM (Jardine, 2006). Indeed, the event data are relevant parts since they enable integration between health assessment of a physical asset with the maintenance actions subsequently decided, the used resources and costs. Therefore, before considering any evolution of CBM thanks to the advancement of techniques for diagnosis or prognosis or IT systems etc, it was deemed important to consider the availability of the CMMS as a basic cornerstone to go ahead. Reasoning by analogy, it is like saying that now, within this work, we are more interested in recognizing if "the building is good" (being based on some very basic elements such as the CMMS) rather than evaluating if "we can live better in the building due to new living solutions therein" (i.e., new technologies for CBM programs). This paper focuses on small and medium enterprises (SMEs) to understand the "quality of their building". An empirical research aims at: (i) understanding whether a CMMS is there or not, and if it is, (ii) which kind of implementation is preferred, and, last but not least, (iii) if this preference is affordable or not for developing advanced maintenance management systems such as those based on CBM programs. The focus on SMEs was selected both because SMEs' high presence in Italy and of their probable lack of economic resources, computer science and maintenance competences, leading to poor maintenance information systems, which may eventually determine a poor "building" where to make CBM live. It should be clear to the reader, since now, that we do not intend to conclude anything about the possibilities of CBM in a SME. In opposition, we intend only to provide empirical evidences of the features of the maintenance information system of SMEs which is, for sure, a constraint for deciding when and how much a CBM strategy is implemented.
\end{abstract}

Keywords: Small-Medium Enterprises, CMMS implementation, Condition Based Maintenance.

\section{INTRODUCTION}

In the recent years the attention to Condition Based Maintenance (CBM) has increased a lot, also due to the improvement in the Information Technologies (IT) solution of the last decades. CBM consists of a maintenance program that recommends maintenance actions based on the information collected through CM (condition monitoring). A CBM program attempts then to avoid unnecessary maintenance tasks by taking maintenance actions only when there is an evidence of abnormal behaviours of a physical asset. Furthermore, CBM, if it is effectively implemented, can significantly reduce the maintenance costs by reducing the number of unnecessary scheduled preventive maintenance operations (Jardine, 2006).

Nevertheless, the establishment of a CBM program regards many issues from both a technical and a managerial point of 
view. Crespo and Gupta (2006) defined a basic supporting structure to enable and to ease the maintenance management process in an organization. This structure is based on three pillars, namely: the IT pillar, the maintenance engineering methods pillar and the organizational (or behavioral) pillar. In respect with the IT Pillar the authors state that a CMMS is a fundamental for the effective maintenance management. CMMS allows to access to the equipment's data and transform these data into information that can be used to prioritize actions and to make better decisions on maintenance activities. The CMMS allows also the proper control of the system and it is more important when the number of the items to maintain and the complexity of the plants are higher. Thus, CMMS seems a success factor for organizing maintenance programs.

The importance of the CMMS arises also in a previous research reported in (Fumagalli et. al, 2008), through a case study analysis, where industries pointed out the adoption of CMMS as a cornerstone of their maintenance activities, before implementing other IT tools. Other works demonstrated the attention to CMMS and CBM paid by the industrial companies (Aberdeen Group 2006).

On the other hand, a survey analysis (Chinese and Ghirardo, 2007), developed on a sample of companies (mainly SMEs) in the Northern part of Italy, stated that even if the $61 \%$ of the steel-making enterprises of the sample adopts a CMMS, only the $15 \%$ of other process industries and the $21 \%$ of manufacturing companies do the same. This research provides also a focus on the adoption of CMMS in respect with the dimension of the enterprise. SMEs adopt CMMS respectively in the $21 \%$ and $29 \%$ of the cases, while the $55 \%$ of the large enterprises adopts CMMS. The lack of diffusion of CMMS in the SME is evident. A similar survey, mainly based on Spanish SMEs (Goti and Arnaiz, 2008), provides a particular result: the $54 \%$ of the enterprises invested in CMMS but only the $45 \%$ really uses CMMS during daily maintenance management. O'Hanlon (2005) finds another interesting issue in his own survey: the $57 \%$ of the enterprises states that the expected ROI (return on investment) for the adoption of a CMMS was not reached. One of the main difficulties in the implementation of CMMS pointed out by O'Hanlon is the needed customization of the CMMS to really adopt it (as stated by the $64 \%$ of the sample).

Thus, if, on one hand, CMMS is declared as a suitable tool to enhance maintenance activities, on the other hand it finds low success, at least in specific industrial contexts.

From the literature analysis, the problem of failure of a CMMS adoption seems particularly present in SMEs. Accordingly to this evidence this paper aims at investigating the sector of SMEs, where to make a snapshot of the AS-IS situation of the adoption of CMMS.

In order to understand which solutions are suitable for SMEs, small and medium producers and sellers of software solutions for maintenance management were interviewed, in order to indirectly derive evidences for their customers. The SMEs, being software producers and sellers will be indicated as SME software provider, while their customers, when SME, will be called SME customers. This is a convention that will be used in the remainder to facilitate the reader. The research was then grounded on two basic questions: (i) are SMEs clients of medium-level / economic CMMS produced by small-medium software houses? (ii) which are the fundamental features required by a SME customer to these low cost CMMSs?

This paper is subsequently structured: paragraph 2 describes general features of CMMS, paragraph 3 explains how the research has been conducted, paragraph 4 comments on the results of the research. Last but not least, paragraph 5 provides concluding remarks, having some specific concern to the links between CMMS implementation and the interests and possibilities to implement a CBM program.

\section{COMPUTERIZED MAINTENANCE MANAGEMENT SYSTEM}

The maintenance management information system is constituted by the data and information (such as an equipment tree, or a meter reading) input to or output from the maintenance activities, by the managerial practices (codified as norms and procedures) used for maintenance management, and by the tools and technologies (such as hardware, operating systems, mobile devices, etc.) used to collect, store, compute and distribute the above mentioned information and documents (UNI 10584/97).

The maintenance management information system can be implemented manually by paper-based documents, as well as by unstructured information organized in general purpose software (such as a spreadsheet). Otherwise, the maintenance management information system can be implemented with specific application software, namely a Computerized Maintenance Management Systems (CMMS) (Wireman, 1994). A CMMS is an integrated set of computer programs and data files designed to provide its user with a costeffective means of managing the massive amount of maintenance data in a standardized way (Cato and Mobley, 2002). Specifically, a CMMS can handle the maintenance transactions the same way an Accounting Information Management System can manage financial transactions. In the case of asset maintenance, the transactions are work orders instead of invoices, and inventories are given by the work's backlog and spare-parts stocks. According to O'Hanlon (2005), common features of a CMMS are: i) Easy Work Order Management, ii) Planning Function, iii) Scheduling Function, iv) Budget/Cost Function, v) Spares Management, vi) Key Performance Indicators (KPI).

Introducing successfully a CMMS should increase, in the long term, the productivity and the competitiveness of the enterprise (Dedrick et al., 2003). Unfortunately, adopting the best-of-breed CMMS software is not an easy task, because more than $300 \mathrm{CMMSs}$ are reported on the software market (see http://www.cmmscity.com), with different technical characteristics and prices ranging from about $800 \$$ to more than 400,000\$ (Braglia et al., 2006). Moreover, the CMMS requirements are not easy to be defined (Swanson, 1997), (Kans, 2008). According to Dunn (1997), most CMMS' implementation projects failed, being the achieved benefits 
noticeably below the company's expectations. According to De Lone and Mc Lean (2003), the following drivers can mostly influence the success of an information system:

- the system quality, that can be evaluated as the system usability (or user-friendliness), availability, reliability, adaptability and time of response;

- the information quality, that can be evaluated by the degree of completeness, easiness of comprehension, customizability, importance and security of the information itself;

- the service quality of the IT department (or external service provider) personnel (reliability, quick response, warranty, empathy with the customer).

These factors can influence noticeably the intention to use and the real use of the system, determining the user satisfaction or dissatisfaction, and finally the net benefits the system can provide (e.g. less resource consumption, time savings, market growth, revenues increase, etc.). These concepts have been used in our research, in order to formulate the factors that determine the adoption/usage of CMMS in SMEs.

\section{RESEARCH SCOPE}

The research has been conducted aiming at retrieving information about the adoption of CMMS. Such kind of researches are normally conducted within an industrial scope, e.g. by interviewing maintenance experts or however industrial managers.

The research presented in this paper, instead, approached the problem by a software house point of view. By interviewing 10 enterprises, being software producers or sellers, information related to about 600 customers was collected. Obviously the information is poor if the purpose is to analyze the business of each single customer. Nevertheless, since one purpose was to understand in which sector CMMSs are diffused, and thus where more attention is paid to CMMS solutions and where there is more work to do, the approach seemed to fit the research goals.

A questionnaire, purposely developed and composed by 24 questions, was sent in an European scope. The response rate was quite low (about 16\%) but enough to achieve a big sample of customers of the software houses. It is worth noticing that the response rate influenced the scope of the investigation, since, at the end, most of the respondents was Italian. The results collected through the survey are showed by tables in the next paragraph.

Some information is also provided here in order to describe the general feature of the sample.

$40 \%$ of the SME software providers that filled in the questionnaire has a number of employees less than or equal to 20 , while $20 \%$ has a number of employees between 40 and 50 . The number of customers for each software house that filled in the questionnaire is reported in the following table 1.
Table 1. Number of customers for each software producer/seller

\begin{tabular}{|c|c|}
\hline Number of customers & $\begin{array}{c}\text { \% of SME } \\
\text { software } \\
\text { providers }\end{array}$ \\
\hline less or equal to 10 & $30 \%$ \\
from 11 to 20 & $10 \%$ \\
from 31 to 40 & $20 \%$ \\
from 41 to 60 & $20 \%$ \\
from 101 to 200 & $10 \%$ \\
from 201 to 300 & $10 \%$ \\
\hline
\end{tabular}

The dimension of the customers (considering, as a whole, all the customers of the SME software providers filling in the questionnaire) is reported in the next table.

Table 2. Dimension of SME customers

\begin{tabular}{|c|c|}
\hline Number of employees & $\begin{array}{c}\text { \% of customers } \\
\text { of SME software } \\
\text { providers }\end{array}$ \\
\hline less or equal to 10 & $1 \%$ \\
from 11 to 50 & $6 \%$ \\
from 51 to 250 & $75 \%$ \\
more than 250 & $18 \%$ \\
\hline
\end{tabular}

So, it is clear that the major part of the clients of the CMMS providers is composed by SMEs, with less than 250 employees.

\section{RESULTS}

The distribution of the CMMS software among the industrial (and non industrial) sectors in the sample of this research is herein presented in the following table.

Table 3. Distribution of the CMMS software among the industrial (and non industrial) sectors. The percentage indicate the percentage of customer belonging to a certain sector. $100 \%$ are the total customers of the entire sample of CMMS vendors.

\begin{tabular}{|l|c|}
\hline SECTOR & \% \\
\hline Automotive OEM & $19,1 \%$ \\
Chemicals & $8,4 \%$ \\
Distribution \& Logistics & $13,8 \%$ \\
Energy industry & $0,6 \%$ \\
Food \& Beverage & $5 \%$ \\
Healthcare/hospitals & $20,9 \%$ \\
Leisure industry, hotels & $0,2 \%$ \\
Mechanic industry & $5 \%$ \\
Pharmaceuticals & $0,4 \%$ \\
Public utilities & $6 \%$ \\
Pulp \& Paper & $1 \%$ \\
Steel making & $2,5 \%$ \\
Textiles & $1,9 \%$ \\
Real estate & $9,3 \%$ \\
Other & $5,9 \%$ \\
\hline
\end{tabular}


A main result is that the healthcare and hospital is a relevant sector that adopts CMMS, thus it represents a good market for the SME software providers. This cannot be considered a good information for the customization of the CMMS toward the industrial needs: the fact that a CMMS producer can be focused also (or mainly) on this non industrial market may induce a potential bias in the offer of CMMS for industrial applications.

Going ahead, sectors that represent high capital industry like cement industry, pharmaceuticals and steel making, are not a large part of the customers of the interviewed enterprises. This is reasonable and can be motivated by the fact that in these sectors the CMMSs adopted are generally provided by big software houses (this is confirmed also by the already mentioned research of Chinese and Ghirardo (2007): since the steel-making companies are the main users of the CMMS, they probably exploit another kind of CMMS market, namely the market of the big producers of software).

Instead, the automotive sector seems to represent the largest part (19\% of the sample), between all the industrial sectors, which is covered by the SME software providers. Indeed, looking at the other customers that adopt low cost CMMS (see again table 3), other industrial sectors are not well covered.

Table 4 focuses on the main features required to the CMMS software as basic preferences. It is clear that the necessity to manage the condition based maintenance (CBM) procedures or the outsourcing services is poorly considered.

Table 4. Percentage of the SME software providers interviewed stating the importance of CMMS features for their customers $(100 \%$ means all interviewees are stating a feature as important; 0 means that no company is stating the importance of a feature for their customers).

\begin{tabular}{|l|c|}
\hline $\begin{array}{l}\text { Main CMMS features } \\
\text { required by the SME customers }\end{array}$ & $\begin{array}{c}\text { \% of } \\
\text { companies }\end{array}$ \\
\hline $\begin{array}{l}\text { Management of planned and unplanned } \\
\text { maintenance }\end{array}$ & $100 \%$ \\
\hline Record of historical data & $100 \%$ \\
\hline Analysis of historical data & $80 \%$ \\
\hline Work orders management & $70 \%$ \\
\hline Control of costs and budget & $70 \%$ \\
\hline Spare parts management & $60 \%$ \\
\hline $\begin{array}{l}\text { Management and control of technical } \\
\text { drawings and documents }\end{array}$ & $40 \%$ \\
\hline $\begin{array}{l}\text { Management of maintenance purchase } \\
\text { order }\end{array}$ & $30 \%$ \\
\hline $\begin{array}{l}\text { Management of condition based } \\
\text { maintenance procedures }\end{array}$ & $20 \%$ \\
\hline Management and control of outsourcing & $0 \%$ \\
\hline
\end{tabular}

As a comment for the empirical evidences reached in the research, it is now worth pointing out that, regarding CMMS, both vertical and horizontal solution exist: henceforth, it is interesting to describe how this offer can be related with the results of this investigation.
Vertical solutions, more properly called industry-specific solutions, are basically offered as products that include sector practices and are customized on specific sector features. These products mainly belong to big CMMS providers that have developed such kind of systems along different projects carried out in a specific industrial sector. After years of experience, then, the CMMS provider that carried out many implementation projects within the same sector has been able to provide a solution dedicated to that sector that requires minor customization for the customer.

On the other hand, SME software providers, if not specialized on an industrial sector, are not able to provide that kind of industry specific solution. They can only manage a general solution (horizontal solution) based on a platform that often requires a great effort to be customized for the sector of the customer, in order to be effective. This is the reason why a great part of the business with the SME customers is related to the consultancy projects in order to: i) introduce the CMMS, starting from the integration of accounting systems and ERPs; ii) subsequently, introduce new maintenance paradigms (i.e. CBM); iii) adopt the CMMS as a means to make these paradigms effective in the customer's plant, through a customization of the product.

The worst situation is when the product, sold by the SME software providers, more than a generic platform enabling customization is a dedicated software tailored for another customer, which is operating in a different sector. Unfortunately this happens and it can be a practical cause of the negative feedback on the CMMS implementation by the customers involved in such kind of situation. The situation is even worse when considering that sometimes SME software providers cannot guarantee a long period of service assisting the customers, due to the limited amount of resources that can be dedicated after first installation, while the implementation of an information system like a CMMS would require a long and complex process, with adequate expenses for its real deployment.

The scarce interest towards CBM expressed by the CMMS providers (on behalf of their clients, see previous table 4) can be motivated by the type of solution offered to the market.

The SME software providers, having less than 20 customers (table 1), try to sell tailored software as a package, replicating their best offers regardless the industry-specific needs and customers requests. It is then difficult that the features they implemented in their products can really enable CBM as a management paradigm. Indeed, SME providers have not enough resources to develop and propose specific solutions tailored for CBM practices.

Besides, other reasons for the scarce interest are in the scope of the research here presented, related both to the needs expressed by the customers and the market dimension.

- There is no need of an application dedicated to CBM management or other issues related with CBM from the point of view of the customers. The SME software providers do not press for this because they are not able to satisfy this need in a complete way. The understanding of the causes that lead customers 
to not understand the importance of CBM (and, eventually, request these features) is out of the scope of this paper.

- A problem of market dimension finally arises from this research. The SME software providers mainly compete on a regional market. Accordingly, they exploit any sales opportunities locally, such the ones offered by the industrial associations, the commercial events promoted by local practitioners' communities, etc. In this local market, companies potentially interested in CBM may be very few; as a result, the returns on investment on the software development of CBM enabling features could not be very attractive.

\section{CONCLUSIONS}

Through the analysis presented in this paper, an evidence of the state of the art of the CMMS implementation in SMEs arises. In particular, the research is clearly bounded by the features of the market: i) SME customers dispersed in lots of sectors and ii) SME software providers probably selling horizontal solutions, since they are not able to gain massive experiences on specific industrial sectors (only the automotive sector, as seen, is the industrial sector with an adequate coverage).

Then, there is the evidence that the recent paradigm of mature maintenance policies are not under development in the SME customers: i) the CBM is not really required as a preference; ii) moreover, outsourcing is not considered, so a market of maintenance experts - which may include also specialists required for CBM - seems out of the preference scope. Even if a development may be on going in some cases (20\% of the preferences in the sample, see tab. 4), it is not as quick as some studies (e.g. Aberdeen Group, 2006) foresee, with main reference to medium-big enterprises. Solutions like CBM are not yet present in many preferences $(80 \%$ does not choose "Management of condition based maintenance procedures" as a required CMMS feature).

This happens even if the technological progress of the recent years introduced on the market tools and solution for data acquisition, data transmission and data analysis that have a relative low cost, so making convenient the adoption also by SME of IT enabling CBM. As depicted by the paper, there is then yet a lot of work to do, in order to enable industrial SMEs to reach a "good building" for further advancement in CBM.

\section{REFERENCES}

Aberdeen Group (2006), The Asset Management Benchmark Report: Moving Toward Zero Breakdown, www.aberdeen.com.

Braglia M., Carmignani G., Frosolini M., Grassi A. (2006), AHP-based evaluation of CMMS software, Journal of Manufacturing Technology Management, Vol. 17 No. 5, pp. 585-602, DOI 10.1108/17410380610668531.

Cato W.W., Mobley R.K. (2002), Computer-Managed Maintenance Systems. A step-by-step guide to effective management of maintenance, labor, and inventory, Butterworth Heinemann, ISBN 0-7506-7473-3;

Chinese D. and G. Ghirardo, Strategie e strumenti di manutenzione nel Nord Est, Indagine sullo stato dei servizi di manutenzione nelle aziende industriali delle Province di Treviso, Pordenone e Udine. MANUTENZIONE, Tecnica e Management - May 2007.

Crespo and Gupta (2006) Contemporary maintenance management: process, framework and supporting pillars. Omega, The International Journal of Management Science, Vol. 34 (2006), pp. 313 - 326

Dedrick, J., Gurbaxani, V., Kraemer, K. L., (2003). Information Technology and Economic Performance: A Critical Review of the Empirical Evidence. ACM Computing Surveys, Vol. 3 No. 1, pp. 1-29.

DeLone W. H., The DeLone and McLean Model of Information Systems Success: A Ten-Year Update, Journal of Management Information Systems, Vol. 19, (Spring 2003), No. 4, pp. 9-30.

Dunn, S. (1997), Implementing a computerized maintenance management system: why most CMMS implementations fail to provide the promised benefits, paper presented at Maintenance in Mining Conference.

Fumagalli L., D. Elefante, M. Macchi and B. Iung (2008). Evaluating the role of maintenance maturity in the adoption of new ICT in the process industry. 9th IFAC Workshop on Intelligent Manufacturing Systems, 9-10 October, Szczecin, PL.

Goti A. and A. Arnaiz, The status of maintenance decision making under economic criteria: survey. On proceedings of 34th Esreda Seminar, Supporting technologies for advanced Maintenance Information Management, San Sebastian, 13-14 May 2008, Spain.

http://www.cmmscity.com

Jardine, A., Lin, D. and D. Banjevic (2006). A review on machinery diagnostics and prognostics implementing condition-based maintenance. Mechanical Systems and Signal Processing, 20 (7), pp. 1483-1510.

Kans M. (2008), An approach for determining the requirements of computerized maintenance management systems, Computers in Industry 59, 32-40

O'Hanlon, T., Computerized Maintenance Management and Enterprise Asset Management Best Practices, 2005, http://www.cmmscity.com.

Swanson, L. (1997), Computerized maintenance management systems: a study of system design and use, Production and Inventory Management Journal, Vol. 38 No. 2, pp. 11-15.

UNI 10584 197: Sistemi informativi per la manutenzione. Italian norm on information systems for maintenance.

Wireman, T. (1994), Computerized Maintenance Management Systems, Industrial Press Inc., New York, NY. 